\title{
Psychiatric outreach clinics held in a general practice setting and community mental health centre
}

\author{
Charles Hindler
}

\begin{abstract}
Over nine months, 57 consecutive newly referred and provious out-patient attenders to two community poychiatic out-patient clinics located in a deprived inner clty area of London were interviewed to elicit demographic information, psychiatric details and obtain their views cobout their preferred location for the out-patient clinic. The majority (94\%) preferred a community out-patient setting to a hospital out-patient clinic, with $76 \%$ preterring a community out-pationt selting to a home assessment. The overall aftendance rate was $89 \%$. A $10 \%$ higher rate of attendance was found at the community mental health centre compared with the general practice and $15 \%$ higher rate for new referrals. Patients preterred to attend a psychiatric out-patient clinic based in the community. This suggests that introducing community-based poychiatric clinics would make a substantial improvement on the current high rates of nonaltendance at hospltal psychiatric out-patient clinics.
\end{abstract}

The development of specialist psychiatric outreach clinics in primary care reflects a coherent strategy of developing communitybased services through the integration of primary and secondary care (Tyrer, 1984). More generally, the provision of specialist out-patient services in primary care has been increasing (Bailey et al, 1994). In particular, with the development of community psychiatric services, general practice based psychiatric clinics have increased in number in recent years (Strathdee et al, 1990) with more psychiatrists running liaisonconsultation clinics in general practice (Pullen \& Yellowlees, 1988). This form of outpatient care was compared with hospital psychiatric clinics, with the majority of patients preferring consultation in the general practice setting (Strathdee et al, 1990).

Hospital-based psychiatric out-patient clinics have been bedevilled by high rates of non-attendance, with figures varying between $28 \%$ to $45 \%$ (Skuse, 1975; Carpenter et al,
1981; Strathdee et al, 1990; Hillis, 1990; Baggaley, 1993). Socio-demographic characteristics of patients have not been found to relate whether they will keep their initial appointment but a previous psychiatric history, referral by a doctor, receipt of a personal appointment letter and attendance at a health centre rather than a psychiatric hospital were factors which have been shown to differentiate attenders from non-attenders (Carpenter et al, 1981; Hillis, 1990).

It has been suggested that outreach clinics in primary care require evaluation with their acceptability to patients as one factor which could be examined (Bailey et al, 1994). Thus far, there has been no research into attendance at such clinics nor whether patient views reflect attendance figures.

The aims of this study were to assess whether patients attending a psychiatric outpatient clinic in a general practice or community mental health centre preferred this community setting to that of a hospital out-patient department or a home visit. Overall attendance rates and the rate at each outreach clinic were also investigated. Based on previous studies (Carpenter et al, 1981; Hills, 1990), an assessment of factors predictive of attendance/non-attendance at hospital out-patient clinics was also undertaken to determine if they were relevant to the community clinics.

\section{The study}

\section{Subjects}

Consecutive patients attending an out-patient clinic based in a general practice venue or a community mental health centre over a period of nine months, were seen by a single consultant psychiatrist $(\mathrm{CH})$. New referrals were randomly allocated by the secretary, 
Table 1. Demographic characteristics

\begin{tabular}{llr}
\hline Mean age & 41.8 years (range 18-72) & \\
\hline Sex: Men & $29(51 \%)$ & \\
Women & $28(49 \%)$ & \\
Mean age leaving education & 18.5 years (range 9-40) & \\
Country of birth & United Kingdom & $36(63.0 \%)$ \\
& West Indies & $6(10.5 \%)$ \\
& Africa & $6(10.5 \%)$ \\
& Europe & $4(7.0 \%)$ \\
& Far East & $2(3.5 \%)$ \\
& Australia & $2(3.5 \%)$ \\
& North America & $1(2.0 \%)$ \\
Clvil status & Single & $47(82.5 \%)$ \\
& With partner & $10(17.5 \%)$ \\
Employment & Employed & $23(41 \%)$ \\
& Unemployed & $33(59 \%)$ \\
Sickness benefit & Receiving & $14(25 \%)$ \\
& Not recelving & $42(75 \%)$ \\
Social class & I & $3(6 \%)$ \\
& II & $13(26 \%)$ \\
& III & $7(14 \%)$ \\
& V & $10(20 \%)$ \\
& Student & $6(12 \%)$ \\
Type of accommodation & Not known & $6(12 \%)$ \\
& Flat/House & 7 \\
Ownership of accommodation & $52(91 \%)$ \\
& Hotel/B\&B/ & $5(8 \%)$ \\
& Hostel & \\
& Private/owned & $13(23 \%)$ \\
& Local authority/rented & $44(77 \%)$ \\
\hline & &
\end{tabular}

blind to the study, to one of the centres. The other patients had attended either one of the venues one or more times in the past. Both the general practice venue and community mental health centre were located in the same deprtved inner city area of South London from which the subjects were also drawn.

\section{Interview}

I devised the interview which covered the patient's demographic characteristics, details of attendance, source of referral, previous psychiatric history, diagnosis, preference for the location of the out-patient clinic and involvement with other mental health professionals of the community mental health team. All subjects seen were interviewed by me.

\section{Statistical analysis}

All btvariate analyses were performed using the $\chi^{2}$ statistic with Yates correction for categorical variables and the Student's $t$-test for continuous variables. Data were analysed on the Statistical Package for the Social Sciences (Version 4.0).

\section{Findings}

Fifty-seven patients participated in the study, 40 attending the community mental health centre and 17 the general practice venue. No patients refused interview.

\section{Demography}

For the sample as a whole. there were almost equal numbers of male and female patients with four out of ten in employment. Twentyone (37\%) were born outside the United Kingdom. The majority lived alone in permanent local authority or rented accommodation (Table 1).

Bivariate analyses of the variables described in Table 1 revealed no significant differences in demographic characteristics between the patients attending the community mental health centre and the general practice venue. 


\section{Details of attendance}

Thirty-three (58\%) attended as new referrals, $21(37 \%)$ to the community mental health centre and $12(21 \%)$ to the general practice. Forty-eight (84\%) patients attended their first arranged appointment with a further three (5\%) attending at a later date after failing to present themselves for the first appointment. Six patients never attended. Hence the overall attendance rate for the two venues was $89 \%$ (51 out of 57) over the nine months.

Thirty-two (56\%) had been referred by their general practitioner, $16(28 \%)$ by a hospital consultant and nine (16\%) from other sources. Forty-three $(75 \%)$ had a previous psychiatric history with 19 (35\%) having a current diagnosis of schizophrenia, $16(30 \%)$ of manic depressive illness, $14(26 \%)$ of neurotic disorder, three $(5 \%)$ suffering with alcohol or drug problems, one $(2 \%)$ having committed deliberate self-harm and one $(2 \%)$ having social difficulties. In three cases the diagnosis was not known but for the other three subjects who had never attended, the diagnosis was elicited from the case-notes.

Bivariate analysis showed no significant differences between the two centres in terms of patients attending and reattending the venues, appearing for their first appointment, source of referral, and previous psychiatric history or diagnosis. Of the 17 patients who visited the general practice venue, 12 attended for their first appointment and two came after a reminder, constituting an attendance rate of $82 \%$ over nine months. Of the 40 patients who visited the community mental health centre, 36 attended for their first appointment and one came after a reminder, constituting an attendance rate of $92.5 \%$ over nine months. $\chi^{2}$ analysis revealed no significant difference between the two centres in terms of these attendance rates.

Bivariate statistical analyses revealed no significant associations between attendance/ non-attendance and the demographic features described in Table 1 , or in relation to place of attendance, first appointment or regular attender, source of referral, previous psychiatric history, diagnosis or involvement with other mental health professionals. To avoid bias from previous clinic attenders on the attendance figures, separate $\chi^{2}$ and $t$-test statistical analyses were conducted on those patients presenting as new referrals in relation to the above described variables. There were no significant findings other than first time referral patients were significantly less likely to be involved with another mental health professional $\left(\chi^{2}\right.$ [Yates] 5.3 ; degrees of freedom $=1 ; P=0.02$; odds ratio $5.5 ; 95 \%$ confidence intervals $=1.2$ to 25 ). As regards the attendance rates of the new referrals, $90 \%$ (19 out of 21 ) attended the community mental health centre whereas $75 \%$ ( 9 out of 12) attended the general practice. This difference was not found to be statistically significant.

\section{Preference for community venue over hospital out-patient setting?}

Forty-eight (94\%) preferred to attend a psychiatric out-patient clinic in the community, two (4\%) favoured the hospital setting, one $(2 \%)$ had no preference and there was no information for the remaining six patients. Bivariate analysis revealed no significant relationship between preferences and the demographic characteristics (Table 1) and no significant associations were found in relation to details of attendance, source of referral, previous psychiatric history, diagnosis, preference for community venue over home visits or involvement with other mental health professionals.

\section{Preference for community venue over home visits?}

Thirty-nine (76\%) opted to attend a psychiatric out-patient clinic in the community but nine (18\%) patients elected for home visiting and three (6\%) showed no preference. There was no information for the remaining six patients. Bivariate analysis revealed no significant relationship between preferences and the demographic characteristics of the patients and there were no significant associations found in relation to details of attendance, source of referral, previous psychiatric history, diagnosis, preference for community venue over hospital out-patient setting or involvement with other mental health professionals.

\section{Involvement with other mental health professionals}

Fifteen (29\%) patients had contact with other members of the community mental health team, $36(71 \%)$ did not and for six there was no information. Of these $15,11(73 \%)$ were seen in a community out-patient setting by the 
team member and four (27\%) were not. All eleven patients were happy with being seen as community out-patients.

\section{Comment}

This study has shown that patients much prefer to be seen in a psychiatric out-patient clinic based in the community than to go to a hospital out-patient department or be visited at home. A $10 \%$ higher rate of attendance at the community mental health centre was found compared with the general practice for all the patients and a $15 \%$ higher rate for new referrals, but this did not reach statistical significance. No differences were found between patients attending the general practice venue or the community mental health centre in terms of patient characteristics, attendance rates and views on attending outreach clinics, suggesting that the service provided in both places was similar and equally satisfactory. The satisfaction with such community clinics also embraced the services provided by non-psychiatrist mental health professionals. This preference for community clinics over hospital-based treatment measured at $94 \%$, closely matched the attendance figure for these clinics of almost $90 \%$. These findings accord with previous work that showed that $83 \%$ of patients attending primary care clinics preferred to attend that setting and the attendance rates at primary care clinics were significantly higher in contrast to a $45 \%$ failure rate to attend a hospital out-patient clinic (Strathdee et al, 1990).

However, almost $20 \%$ of patients would have preferred to be seen at home as opposed to attending a community psychiatric clinic, indicating that such clinics are not necessarily ideal for all patients. It was not possible to differentiate this former group demographically or psychiatrically from those favouring the clinic setting. Nevertheless, locating a community psychiatric teambase within a catchment area and running an out-patient clinic from this or a general practice, or both. would allow for increased flexibility to provide both an out-patient or home-based service. It would also facilitate greater accessibility between primary care and the psychiatric service (Tyrer, 1985).

Factors predictive of attendance/nonattendance at hospital out-patient clinics were not found to be predictive for community clinics, indicating that further work is required. To elicit such factors as this could reduce the waste of health care resources consequent on patients failing to arrive at the clinics. Nevertheless, it appears from this research that introducing community-based psychiatric clinics would make a substantial improvement on the current high rates of nonattendance at hospital psychiatric out-patient clinics.

Criticisms of this study are that the interviews were conducted by the treating clinician, which could have biased patients to provide socially desirable responses or reflected an unwillingness to disappoint the interviewer. Secondly, the inclusion of previous out-patient attenders might be viewed as unhelpful as their very presence suggested that they were satisfied with the out-patient treatment. However, they acted as a comparison group for the first-time attenders with no statistical differences found between the groups. It has also been shown that some of the previous attenders did not regard the community-based out-patient clinic as the most preferred place to be seen, lending further credence for their inclusion in the sample. Finally, the patients studied were only attending a community facility. A sample attending either a hospital or community clinic, or receiving treatment at both centres could have strengthened the methodology of this study.

\section{Conclusion}

Community-based out-patient clinics are experienced by new and regular attenders as being better than hospital-based clinics and home visits, as shown by the high attendance rates and positive response to these clinics. Reasons for this remain unclear at present and should form the basis for further research.

\section{References}

BAGGALEY, M. (1993) Improving the attendance for new psychlatric out-patient referrals. Psychiatric Bulletin. 17. 347-348.

BAnEY, J. J., BLACK, M. E. \& WILKIN, D. (1994) Specialist outreach clinics in general practice. British Medical Journal, s08, 1083-1086.

Carpenter, P. J., Morrow, G. R. Del Gaudio, A. C. \& RrtzLER, B. A. (1981) Who keeps the first outpatient appointment? American Journal of Psychiatry. 138. 102-105. 
HiLus, G. (1990) Rejection of psychiatric treatment. Psychiatric Bulletin, 14, 149-150.

PULEN, I. M. \& YeuowleES, A. (1988) Scottish psychiatrists in primary health-care settings: a silent majority. Brttish Journal of Psychiatry. 153, 663-666.

SKUSE, D. H. (1975) Attitudes to the psychiatric outpatient clinic. British Medical Journal, 3. 469-471.

StrathdeE. G., KInG. M. B., Araya, R., et al (1990) A standardized assessment of patients referred to primary care and hospital psychiatric clinics. Psychological Medicine, 20, 219-224.
TYRER, P. (1984) Psychiatric clinics in general practice: an extension of community care. British Joumal of Psychiatry, 146, 9-14.

- (1985) The hive system. A model for a psychiatric service. British Journal of Psychiatry. 146, 571-575.

Charles Hindler, Research Fellow in Psychiatry, The Royal Free Hospital, Pond Street, London NW3 2QG 